\title{
Information Technology in NHS Direct using Wireless Mark-Up Language (WML) Environment
}

Peter Okpamen

\author{
Ambrose Alli University, \\ Ekpoma-Edo State, Nigeria.
}

\section{Doi:10.5901/ajis.2013.v2n6p103}

\begin{abstract}
National Health Services (NHS) Self-help guide is simply an application of mobile phone in a platform independent (WML) environment to sort out solutions to health symptoms. Essentially, the success of this project goes a long way in simplifying the pressure on the already stretched services in the NHS, London. The project is principally based on the openwave technology which enables such wireless platform independent services. The service provides an opportunity for the user to access the 'search page' in the network, and identify the symptoms he or she has; thereafter deal with the questions in the 'question card'. Application of this model no doubt has helped in dealing with the pressure faced by the health services of NHS, London. It is imperative therefore, for developing countries and other organizations to take advantage and invest in the enabling technologies in place, in order to deal with the huge pressure faced by health institutions; as well as help in dealing with other forms of conflicts in organization.
\end{abstract}

Keywords: Information Technology, Mobile Phone, NHS (National Health Service), Switchboard, Symptoms, Management, WML (Wireless Mark-up Language).

\section{Introduction}

The main task in this project is to design and implement a mobile application based on NHS SelfHelp Guide. The WML (wireless mark-up language) environment and the Openwave technology (openwave refers to the company that provides the application infrastructure for wireless data), will be used to create the application. The advent of technology has made this application possible, and in the case of a mobile phone technology it is unique in the sense that its application is platform independent. In order words, the user could be anywhere, and as long as the network system is in place the user can always adopt the NHS Self-Help Guide. This is as a result of the breakthrough in the world of Information Technology.

Information Technology has been a blessing to modern day organization because of its immense benefits to the organization. The beauty of the mobile application technology is in its feature of platform independent. The use of the mobile application in the NHS Direct model appears to be the fastest way for health managers to have quick access to the problems of the numerous patients out there, irrespective of where the patient may be at any particular time.

Resolution of the symptoms if not complete can be achieved tentatively. So it is necessary for management in other organizations to develop such model and encourage people in organization to imbibe the use of this model in order to deal with pressure within the system. Management therefore owe it a point of duty to make sure the system is secure in terms of identity protection, as well as making sure the system is effective and efficient at any time. In the past, quick access to the consultant is not possible because of the absence of the mobile system. 
However, the application involves a few stages and also requires the user to know a simple practical application on how to operate the mobile phone to achieve the desire objective. Knowledge of the application codes is not necessary for the user in this case.

\subsection{Objective of the project}

The basic objective of this project is to demonstrate the application of mobile application to NHS Self-Help Guide. As a result of the impact of this technology, NHS patient can seek solutions to their basic problems without necessarily going to the hospital to queue up waiting to see a consultant. This is also a direct challenge to other organizations to explore the benefits of such mobile application for the overall development of the organization.

\subsection{Significance of the research}

The need to reduce the pressure from patients attending the NHS daily cannot be over emphasized; and this has motivated the desire for this project. The application of Self- help guide provides the relevant advice to the user before deciding on whether to contact the NHS Direct or to dial 999 for emergency. It is therefore imperative that this project will be of immense benefit to all concern users of NHS and other organization that care to emulate such application.

\subsection{Limitation of the Project}

The obvious limitation of this project boils down to the fact that, it cannot be used in an environment without the enabling technology; especially in less developed countries. Furthermore, the knowledge of technology and platform independent is not available in such environment. In London where this project was developed, the system is highly efficient and resourceful to the users. However, Information technology revolution did not help matters as it was purely in favour of the "G7" who solely designed it without necessarily putting into consideration the place of the poor nations that are disadvantageous in the information technology world. Attempting to use this application in these poor countries will obvious lead to an investment in frustration. (Capra, 2002. pp.139).

\section{Mobile Application Technology in NHS:}

The Oxford Advanced learners dictionary, $8^{\text {th }}$ edition, defines Information technology as electronic equipment such as computer for the purpose of storing, analysing, and distribution of words, figures and pictures. Its application in an organization has therefore created greater improvement in terms of efficiency, ability to work without time or resources wasting, information communication and management; as well as helping in the process of evaluating and control within the organization. The advent of Mobile Application in the NHS structure therefore, has actually transformed the organization into a digital health institution. Its application in NHS has therefore provide management with a measure of problem solving such as symptoms management, increase in quality of production, increase in speed of processing, improvement of patients services, enhanced communication and collaboration, etc. The use of mobile application has become a critical success factor in the operations of this organization, especially in the area of NHS Self-Guide that has reduced the level of pressure in the system. Information Technology no doubt has led to major improvement in human ability to collect, process data and disseminates information. With the advent of the internet and the World Wide Web, Information Technology is better felt in organization in recent times. Telecommunication infrastructure, coupled with massive growth of 
personal computers and mobile phone devices provide vast amounts of information to users at any time. In addition to the above, the improvement in advanced information technology has led to a platform for human progress. Amongst the benefits of technological breakthrough include cheaper products resulting from data transformation. Also it is much quicker to access information, data storage, data manipulation, retrieval of data and information, and transfer of data more quickly and effectively.

The advent of advanced information technology, coupled with positive improvement has made global economy somehow like a ghost, cyber-linked, and projected towards a high level of success. Although copra is of the view that information technology has further compounded workers' stress in the organization. As a result of improvement in modern technology, work can now be carried out much faster than before, and more time is now being saved. The idle time on the other hand is now channelled into more avenues by way of making the workers to do more work in order to generate more profits for the owners. In addition, "it is evident that the various key components of today's business environment such as the global competition, corporate mergers, turbulent markets, increasing workloads, "24/7" accessibility via e-mail/cell phones etc, have all constituted a highly stressed and unhealthy situation" (Capra, 2002 .P.127).

Although, the world of information technology that presented the whole world as a global village is now under serious scrutiny by way of information and access control management. Despite all odds in the business arena there has been argument for business success without constraints, in areas like health, education, capital, communication, consumers, corporations, etc (Ohmae, 2005.p.20). Apart from IT affecting the structure of the organization, it has also created a lot of benefits to the organization especially in the aspect of solving health problems and management of problems within and outside the organization. The level of pressure in the system these days appears to have reduced when compared to the past. Things are better organised in the organization now and detection of symptoms and subsequent resolution to these symptoms is faster and more accurate. In terms of competitive edge, the involvement of IT has helped the NHS to gain a wide range of competitive advantage over others in the same line of business. The design of creative applications such as this model has paved the way for NHS to compete more effectively in the health industry. Another traditional benefit of this application to NHS is that, it has helped in the reduction of cost in terms of operations and in terms of dealing with patients. The application has also helped in the automation of clerical task geared towards reducing cost.

\section{Methodology}

\subsection{The Mobile Application}

Essentially, the application is simple to adopt as it does not require expertise knowledge of the use of mobile phone. The only technical aspect is the code for the application which is of no relevance to the ordinary user; but for the purpose of this project the codes are included alongside the application with detail explanations of the necessary aspects. The mobile application involves a few steps to be carried out by the user.

The first stage contains a switchboard which contains a simple program version/code on how the user can get started. On assumption of the code is a form known as the openwave environment. This automatically takes the user to the "Hello" page as displayed on the mobile phone below. The first thing you observe on the screen is the NHS Direct logo and contact information space. The link therein is there to enable the user access to the application. The subsequent second page of the application is arrived at through a click on the select button.

The next stage is the search stage page. It presents before the user a list of diseases classified in letters of English alphabet. On the basis of this the user selects an alphabet indicating 
the symptom. In order words, click on the letter to find out your symptom(s). For example, click on F- for Fever; or click on C-for Cough.

The next stage that follows is the question card which displays written questions to the user based on the disease that he/she chose. The code used for this card is presented in the later part of this project. Here you will be asked some simple questions, and you are expected to select answers to the questions. For example;

NHS Operator: - Do you have a severe headache?

USER: - YES/NO.

Based on the interaction, coupled with your answer, the NHS Nurse will advise you on whether you need medical attention, and if you do, how quickly you should get help before ringing NHS Direct or 999. In line with the above development, if the user's answer to the question asked is YES, Card 2 would be displayed with a picture to tell the user to phone an NHS Direct nurse. On the other hand, if the answer from the user is NO, then the NO card would be displayed advising the user to undertake a self care as it is safe to manage this problem yourself.

\subsection{Implementation of the Switchboard}

The switchboard was designed to provide introduction to the user who wants to use the application. This is the code used for this purpose. The switchboard enables the user to enter the openwave environment, and this contains a display of the NHS logo and a link to help the user access to the application. Usually, the user is expected to simply click on select to go to the next page.

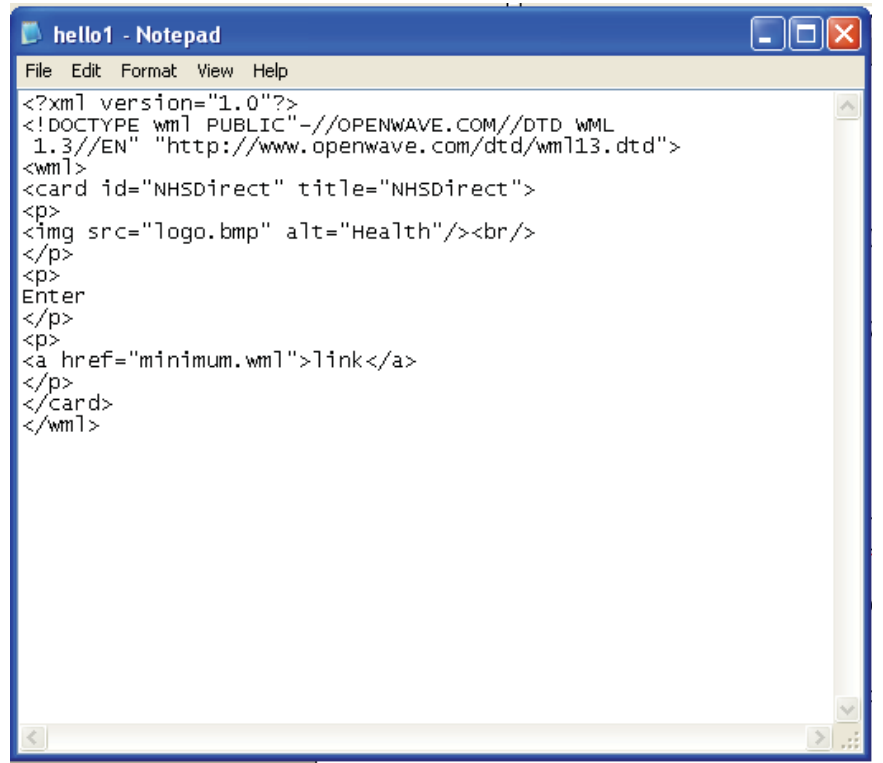

Here is how it looks like in the Openwave environment. 


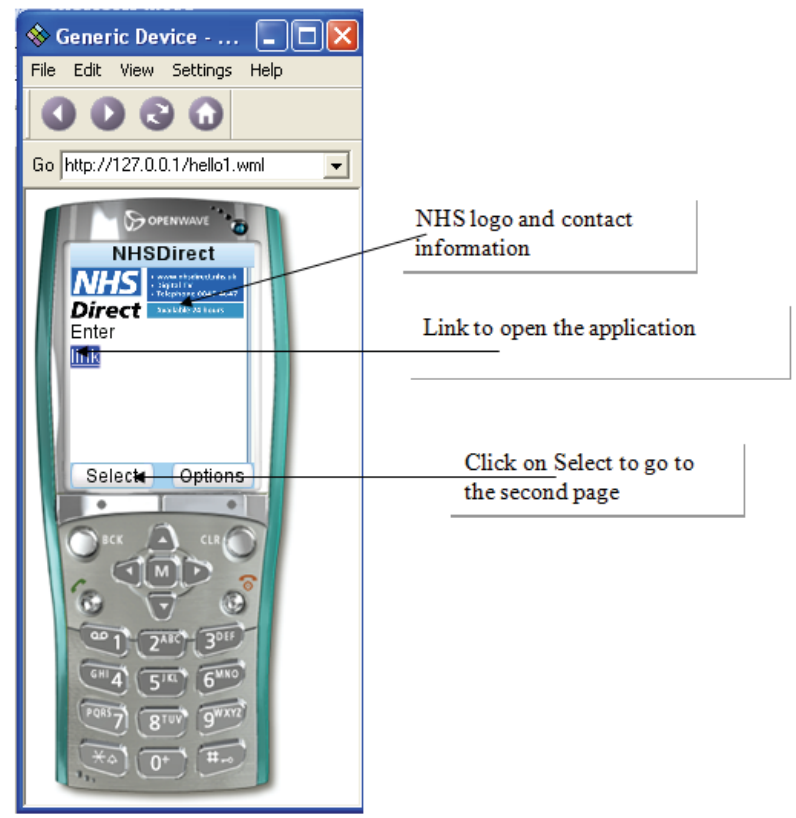

\subsection{The search page}

The Search Page enables the user to choose what disease he thinks that he/she has based on the symptoms. Generally the user can choose from a list starting with letters of the English alphabet, and then the symptoms would be displayed afterwards.

In the program, if the user choose click on the soft button then another card would be displayed showing a choice of diagnosis. At this stage the user is faced with a huge volume of information to choose from. The important thing is to make the right choice of letter indicating the symptoms he/she has. Below is the set of codes presented in the search card below. 


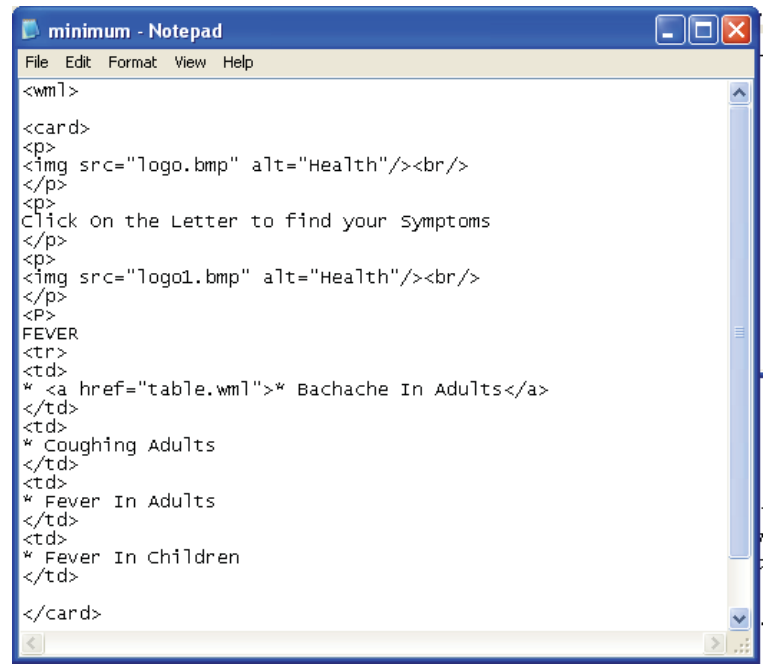

Here is how it looks like in the Openwave emulator.

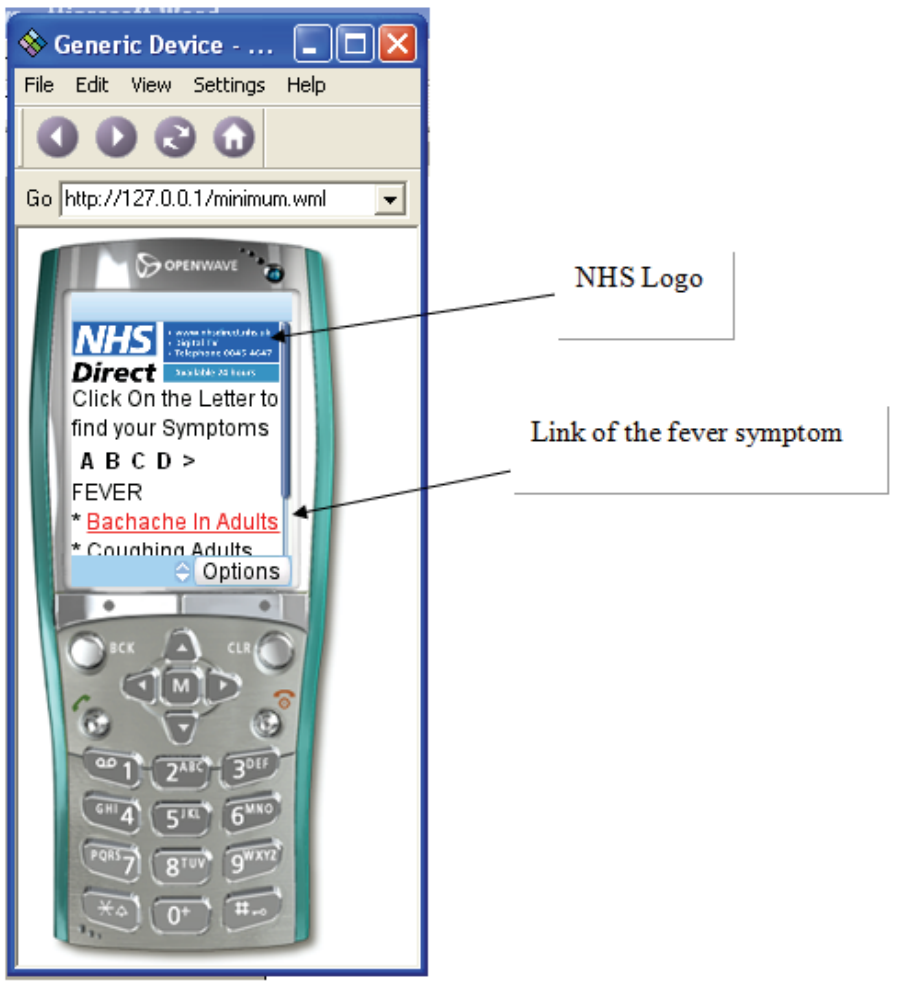

\subsection{The question card}

This card displays a written question to the user based on the disease that he/she has chosen. The user is expected to give the right answer in order for the NHS Self-Help Guide to have useful value 
for the user. For instance; is the fever severe? The user is expected to give a YES or NO answer. A YES answer will lead the user to another stage of the card, while a NO answer could direct the user to adopt a self solution as the symptom is not a serious type to warrant a call to the NHS nurse or 999. Below is a set of codes displayed in the question card.

The code for this card is:

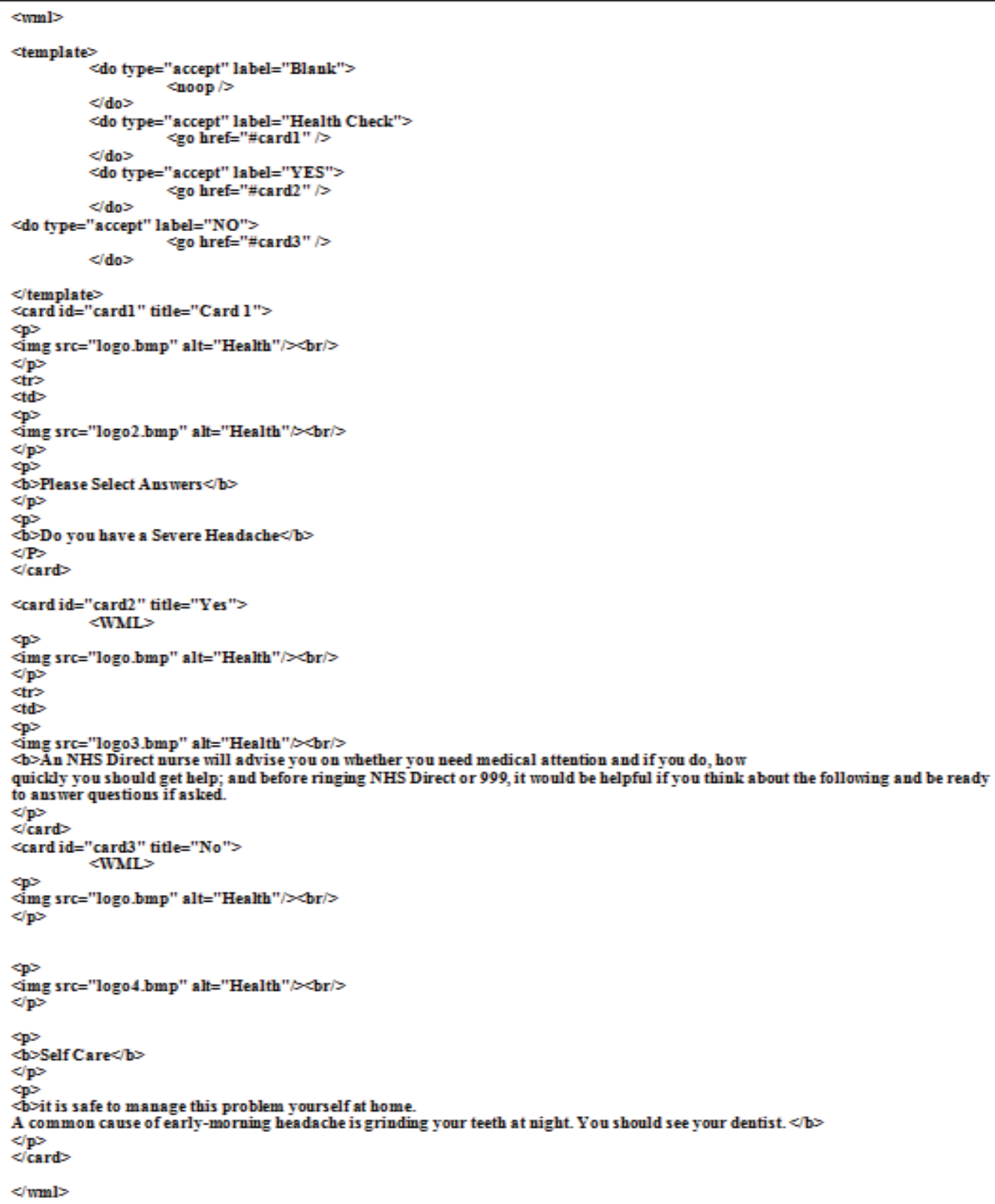


Here is how it is displayed in the emulator.

Here is the outlook of the above code on the openwave emulator.

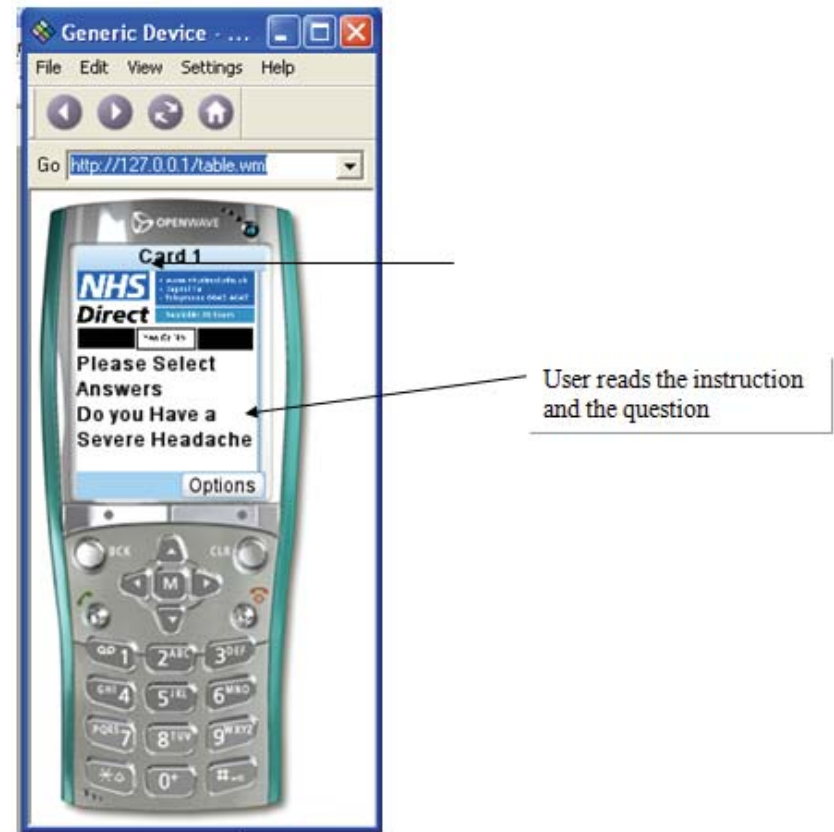

Here is the outlook on the emulator

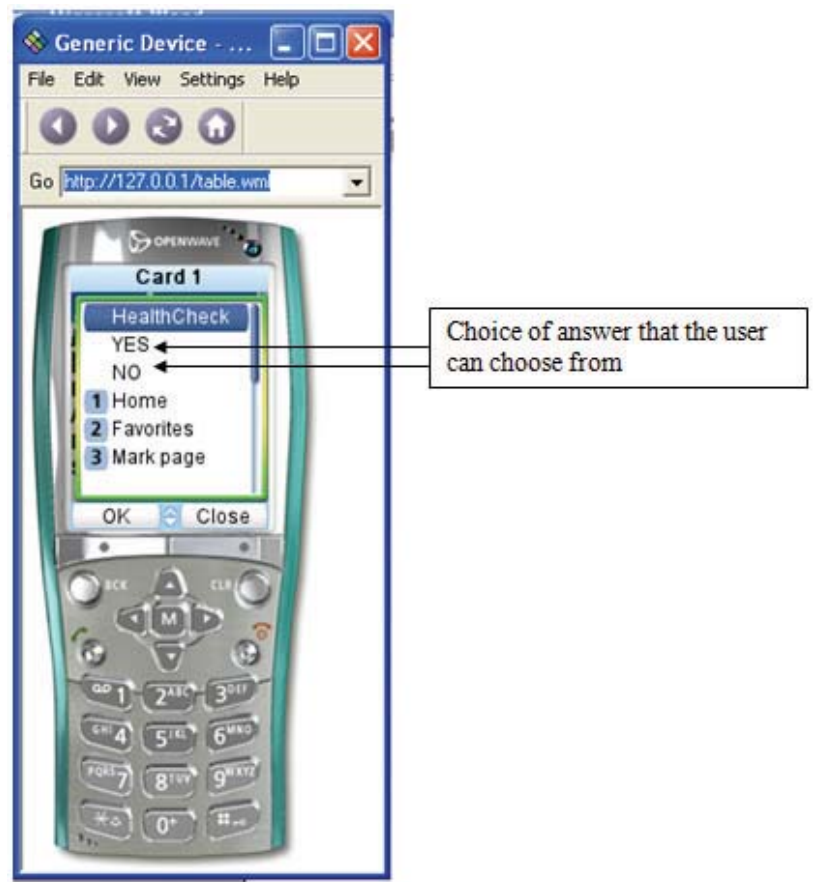


If the user chooses YES then Card2 would be displayed. Here is how it looks.

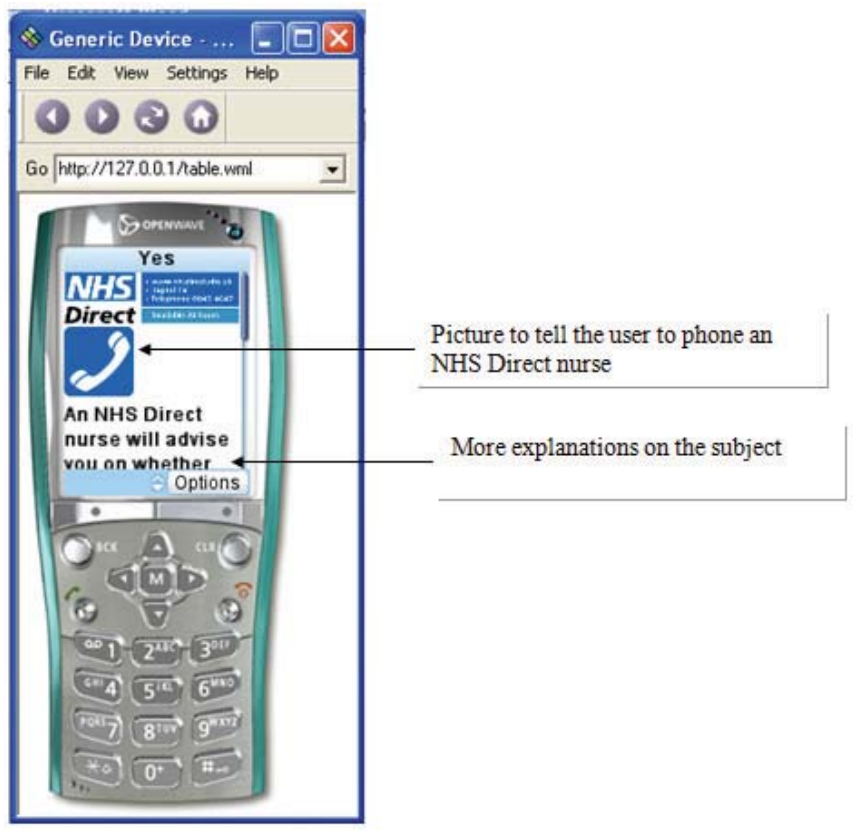

If the answer of the user is No, then the NO card would be displayed.

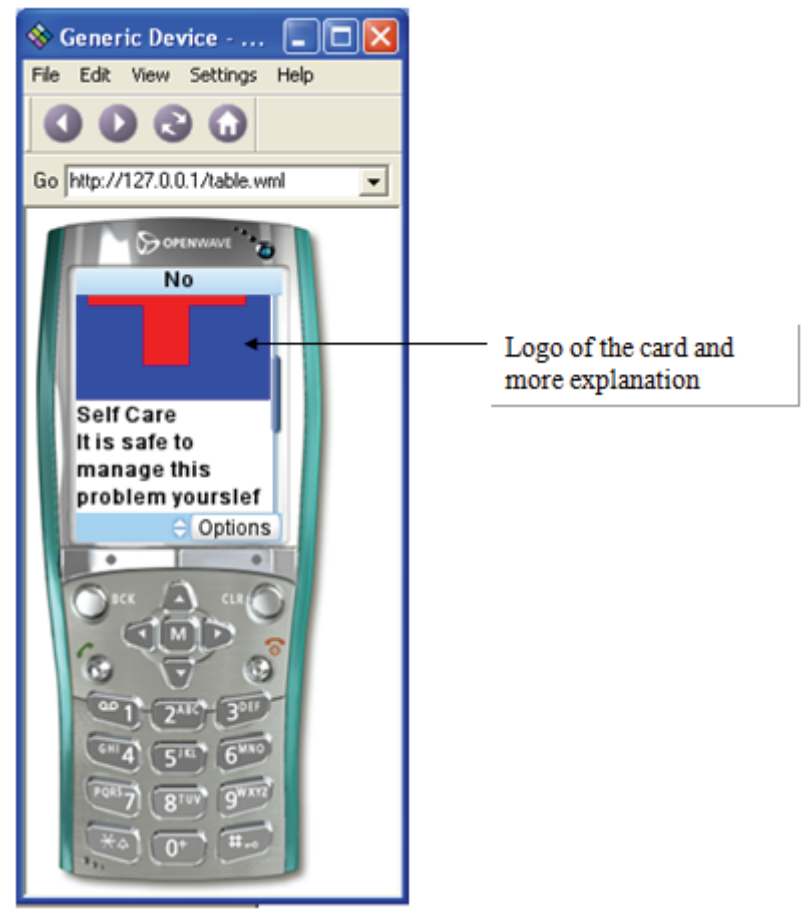




\section{Concluding Remark}

Application of NHS Direct has made life easier for users seeking to adopt the NHS Direct Self-help project, because oftentimes there are queues here and there in hospitals which eventually lead to frustration amongst the patients. Advent of technology and the openwave environment has enabled a mobile application in a platform independent (WML) environment. It is therefore imperative to extend this type of application to other organizations to help deal with other forms of crisis in the organization.

\section{References}

Capra, .F. (2002); The Hidden Connections. First edition; published by Doubleday, 1540, Broadway, New York, New York 10036.

Ohmae, k. (2005). The Next Global Stage. Newjersey, Wharton school publishing.

Oxford advanced learner's dictionary, 8th edition. By A .S, Hornby - Oxford University Press (2010) Paperback - 1796 pages - ISBN 019479900X

http://encyclopedia2.thefreedictionary.com/Openwave

http://encyclopedia2.thefreedictionary.com/Openwave+Mobile+Access+Gateway 